se caracteriza por su faltá de conexión $(89,91 \%)$, lo cual provoca una desestructuración entre dichos conceptos. También el elevado número de errores conceptuales.

2. Los profesores muestran una decidida tendencia a aceptar otras propuestas alternativas que intenten establecer una continuidad entre los temas del átomo y el enlace.

3. Como consecuencia de esta forma de introducción del átomo y el enlace, los alumnos no aicanzan los niveles que podríamos considerar básicos para su aprendizaje y consiguiente formación.

Por ejemplo, respecto a los resultados de tos cuestionarios de los alumnos, son datos destacables que todos los alumnos encuestados de $2^{\circ}$ de BUP y el $76,9 \%$ de los alumnos de $3^{\circ}$ de BUP y de COU desconocen que la única causa del enlace son las fuerzas electromagnéticas, y el $100 \%$ de los alumnos de $3^{\circ}$ de BUP y de COU no reconocen ninguna de las posibles representaciones del átomo y las moléculas.

En general, podemos afjrmar que la introducción de la física moderna no se elucida conceptualmente, sino que se cometen graves errores conceptuales, mezclándose los modelos clásicos y cuánticos, especialmente en el tema del enlace, cuyos modelos se presentan desglosados y sin que se presenten sus límites de aplicación, además de no explicar generalmente las propiedades a partir de la física cuántica.

También se desprende de este trabajo, el interés tanto de la posibilidad de realizar un análisis más exhaustivo de los problemas que presenta al alumno la enseñanza habitual de los conceptos del átomo y sus enlaces, como la posibilidad de crear y desarrollar alternativas que permitan un estudio más crítico de dichos temas.

Estas alternativas se enmarcarían dentro de un enfoque constructivista, tanto desde el punto de vista metodológico como conceptual, abordándose la posibilidad de presentar una visión unitaria del enlace (a partir de un planteamiento cuántico en el nivel superior) y de mostrar además una continujdad manifiesta con el tema del átomo.

\title{
RESEÑAS DE CONGRESOS
}

\section{LA I CONFERENCIA INTERNA- CIONAL SOBRE EDUCACIÓN Y FORMACION EN GEOCIENCIAS}

La conferencia que reseñamos surgió de la frustación de la Sección de Enseñanza del penúltimo Congreso Geológico Internacional, celebradoen Washington en 1988. El escaso número de comunicaciones allí presentadas reveló un desinterés tan preocupante de la profesión geológica por su faceta educativa que se decidió convocar una conferencia internacional específica sobre el tema. La Universidad de Southampton en Inglaterra fue la sede. en abril pasado, de este primer experimento.

Un primer éxito del experimento fue de asistencia: 240 asistentes de 48 países. Lógicamente, una gran mayoría (casi un tercio) de británicos, y tambien, sorprendentemente, una gran representación asiática $(23 \%)$, especialmente de la India. Débil participación, en cambio, de Estados Unidos ( $5 \%$ ), y menores aún de Japón, Francia, Italia, Alemania y España ( 2 asistentes de cada país). Todos fueron alojados en las residencias estudiantiles de la Universidad de Southampton, un delicioso campus para 7.000 estudiantes.
La estructura del Congreso fue la clásica en las reuniones geológicas: excursiones, ponencias y comunicaciones, éstas organizadas en cuatro secciones temáticas: niveles primario y secundario, nive? universitario, la formación del geólogo profesional y la divulgación de la geología al gran público. De forma muy británica, el campo precedió a todas las sesiones: entre otras opciones, un agradable paseo por las formaciones de creta de la costa Sur de Inglaterra. Entre las conferencias introductorias, la de Jeremy Leggett, director científico de Greenpeace, que resultó ser geólogo, fue probablemente la de mayor impacto: el efecto invernadero y la energía para el futuro fueron los temás en los que más se insistió, y con datos sobrecogedores. Sin embargo, para muchos quedó claro que éste es, hoy por hoy, un problema sin solución clara. En lo que no hubo discusión fue en la repetida afirmación por parte de J.L. de que las geociencias deben ser holísticas, y que ello implica el (muy difícil) abandono de la compartimentación que se observa en los actuales planes de estudio, con la peligrosa extensión de la superespecialización en los currículos desde los niveles universitarios hacia los no universitarios.
Entre la media docena de ponencias invitadas merecen destacarse dos: las de Derk Hodson (Ontario, Canadá) y Victor Mayer (Ohio, EEUU). El doctor Hodson arremetió contra las prácticas de laboratorio en la enseñanza de la geología, tal y como están planteadas en el mundo anglosajón (donde pueden ocupar el 50\% del tiempo de clase). Según él, existe una obsesión por "ir al labo" que ni la pedagogía ni las encuestas justifican: simplemente, los alumnos van al laboratorio para adquirir unas habilidades que sólo sirven para trabajar en el laboratorio. Propuso emplear más bien otras alternativas docentes, como ver o hacer vídeos, debates, entrevistas, leer o comentar noticias de prensa, resolver problemas, construir hipótesis... la charla fue viva y provocadora, aunque tuvo dos peros desde nuestro punto de vista: el primero, la digresión no justificada de ataque a los científicos; el segundo, que la filípica contra las prácticas no es extrapolable a este país (ni seguramente a otros muchos), donde simplemente no se hacen.

La charla del profesor Mayer fue mucho más constructiva: ni más ni menos, abogó por una enseñanza científica integrada 
centrada en cl planeta Tierra, idea nuclear en el proyecto Earth \$ystems Project, actualmentc en discusion en Estados Unidos. Se trata de una idea basada en gran medida en la teoría de sistemas, en la que los subsistemas de la Tierra son el punto de encuentro de los procesos físicos, químicos y biológicos a comprender en el currículo de secundaria.

En las comunicaciones, como siempre sucede en estos encuentros, hubo de todo: desde el profesor universitario británico que habia desarrollado un programa de ordenador para decidir la ocupación óptima de su laboratorio de microscopia, hasta el profesor viemamita que leía en trabajoso inglés el plan de estudios de la única universidad de su país donde se puede estudiar geología.

Los ejemplos no están elegidos al azar: tambien en la enseñanza de las geociencias hay un abismo entre Occidente y el Tercer Mundo, con los países latinos, europeos y americanos (Brasil, España, Italia, Portugal, Venezuela, especialmentc) en un aceptable término medio.

En la sesión de clausura, los encargados de las cuatro sesiones presentaron sus respectivas sintesis, de las que destacaremos tan sólo algunos puntos:

- El alumno debería tener opción a aprender lo que le interesa, más que el profesor a enseñar lo que quiere.

- En la universidad, los cursos de mate rias geocientíficas deben cambiar oe ser cursos de conocimientos a cursos que promuevan habilidades. Las clases deben ser mas inspirativas que ins truetivas.

- La apreciación del medio ambiente como un valor global (planetario) debe ser esencial en la enseñanza en ciencias de la Tierra, ahora y en el futuro: los enfoques holísticos centrados en las ciencias medioambientales recibieron un apoyo entusiasta frente a los enfoques reduccionistas que primaban hasta hace bien poco.

- El trabajo de campo plantea problemas especiales, de tipo económico, numérico y legal. Se habló de trabajo de grupo y de megacampamentos multiuniversitarios, pero tambien de que los estudiantes reatizasen su trabajo solos y los profesores los acompañasen después.

- Debe fomentarse la cooperación entre asociaciones de enseñanza de las ciencias de la Tierra como NESTA, NAGT, ESTA, AEPECT (de la cual se sugirió que sirviese de vehículo a los docentes del ámbito latino, tanto europeo como americano), entre ellas y con asociaciones de docentes de otras materias.
- Una estructura internacional como la reunida por vez primera en la conferencia tiene la potencialidad para mejorar la situación en todos los campos citados. Como dijo Chris King (vicepresidente actual de la ESTA), tenemos los datos y tenemos la decisión. Sólo falta ponerse a trabajar.

$Y$ tambien destacaremos algunos interrogantes:

- Los temas de ciencias de la Tierra contenidos en los curriculos preuniversitarios, $¿$ deben estar concebidos para formar ciudadanos cultos o geólogos potenciales?

- ¿Cuáles son las ventajas y desventajas de los currículos nacionales, como los que existen oestán en desarrolloen EEUU, España, Gran Bretaña, Corea y Japón?

El congreso cuido mucho los aspectos sociales. Aparte de las recepciones al uso, hubo dos veladas inolvidables: una a bordo de un jazz-boat por el estuario del rio, hasta la isla de Wigth, y la otra en un pub con una orquestina, inolvidable Wild Orchid, donde uno de los firmantes (J.L.) recibió el segundo premio del concurso fotografico por una foto de la Ciudad Encantada de Cuenca, con el aplauso arrollador del mundo latino.

Es probable que la próxima reunión se celebre en Bulgaria en 1995. Entretanto, uno de nosotros (F.A.) ha sido invitado a ser corresponsal en España de una newsletter que será editada por John Carpenter, de la Universidad de South Carolina, EEUU, con la obligación de enviar al menos una vez al año un informe sobre acontecimientos relacionados con la enseñanza de las geociencias. Este boletín permitirá intercambiar idcas, experiencias y material entre profesores de ciencias de la Tierra en todo el mundo: conexión que es precisamente uno de los objetivos de la AEPECT (Asociación Española de Profesores de Ciencias de la Tierra) desde su fundación.

Francisco Anguita José Lillo
XIX CONGRESO IN'TERNACIONAL DE HISTORIA DE LAS CIENCIAS

Entre los días 22 y 29 de agosto de 1993 se celebró en la ciudad de Zaragoza el XIX Congreso Internacional de Historia de la Ciencia, auspiciado por la UNESCO y que tiene lugar cada cuatro años en un país diferentc. La organización de este acontecimiento corrió a cargo de la Sociedad Española de Historia de las Ciencias y de la Técnica, una entusiasta corporación de investigadores en la historia de muchas disciplinas naturales y sociales que, entre otras cosas, publica la revista Llull. El hecho de que este congreso se hay a celebrado en España denota el momento pujante de la historia de las ciencias en nuestro país, pese a la escasa presencia en los medios universitarios y el poco apoyo institucional.

Dentro del marco de la ciudad de Zaragoza (que hizo amplio eco del congreso a través de los medios de comunicación social y la publicidad en calles y autobuses) se congregaron más de $1.300 \mathrm{con}$ gresistas de 75 países en las amplias instalaciones de la Facultad de Medicina. Como dato anecóótico es necesario resaltar que el dibujo original del cartel fue realizado por el profesor de Moscú Gyorgi S. Kará-Murzá, una de las más prestigiosas figuras de la historia de las matemáticas.

\section{Estructura y desarrollo del congreso}

Durante el congreso se presentaron unos mil trabajos de investigación originales, cuyos resúmenes ocupan cinco gruesos volúmenes. La lectura de los trabajos se realizó dentro del marco de 60 simposios y 75 sesiones científicas. Así mismo, en la Facultad de Medicina -sede del congreso- tuvo lugar una amplia y selecta exposición bibliográfica con 700 novedades exclusivas. También, en la librería Pórtico se expusieron más de 5.000 títulos de filosofía e historia de las ciencias y de las técnicas, y se ha editado un valioso catálogo bibliogxáfico muy bien presentado y que representa un hito importante por su actualización y dimensiones.

Es necesario resaltar el valor de las conferencias impartidas por especialistas durantc el congreso. Resaltamos por su incidencia las siguientes:

- Une science baroque?, a cargo del profesor Jean Dhombres (CNRS) que ocupó el lugar de conferencia inaugural del congreso.

- Development of lasers, fue una de las confercncias estrella, debido a que fue 
impartida por el premio Nobel de Física, Dr. Alexander M. Prokhorov.

- Problemes methodologiques de thistoriographie des sciences, conferencia pronunciada por el profesor Lubos Novy, de la Academia Checa de Ciencias.

- Al-Mut'taman Ibn Hud, King of Saragossa and brilliant mathematician, a cargo del profesor Jan P. Hogendijk, de la Universidad de Utrech.

- L'Utistorie des sciences comme projet intelletuel, conferencia a cargo del Dr. Vicenzo Capelletti, del instituto de la Enciellopedia Italiana.

La conferencia de clausura fue pronunciada por el profesor Mariano Hormigón, de la Universidad de Zaragoza y presidente del congreso sobre la Ciencia, ¿una herramienta liberadora?

\section{Simposios y comunicaciones}

Es difícil resaltar en una breve crónica los hitos y aportaciones más importantes de los simposios temáticos y las comunicaciones. De todas formas, si se considera el interés de los mismos en función del número de asistentes y número de intervenciones, resaltaremos los siguientes simposios:

- Entre la mecánica y la arquitectura.

- Matematización de las ciencias biológicas, economicas y sociales.

- La formación de escuclas matemáticas en los siglos $\mathrm{x} x \mathrm{x}$ y $\mathrm{xx}$

- Las matemáticas en Asia

- Historiografía de la historia de las matemáticas.

- Historia de la geografía en los siglos XIX $y$ XX

- El más antiguo astrolabio latino

- La exploración botánica del Nuevo

Mundo en el siglo xvia.
- Fundamentos de la bioquímica moderna en los siglos $\mathrm{x} x \mathrm{x}$ y $\mathrm{x}$.

- Biología, incluyendo los enfoques químicos y físicos, entre los siglos xvm y $\mathrm{xx}$.

- La minería en América Latina: intercambio de tecnología entre Europa y Latinoamérica.

- La circulación de las ideas científicas en el campo de las ciencias exactas, en el islam medieval entre Oriente y Occidente.

- La estructura internacional de los inter. cambios científicos.

- Las instituciones científicas ibéricas y latinoamericanas.

- Estrategias para el mantenimiento de la enseñanza de la historia de las ciencias

Las comunicaciones científicas tuvieron un carácter temático tradicional de tipo disciplinar, sjendo las más concurridas las de matemáticas, biología, medicina y tecnología

\section{Historia de las ciencias y enseñanza de} las ciencias

Desgraciadamente hubo escasísimas (que sepamos) aportaciones relacionando la historia de las ciencias y las técnicas con la enseñanza de las ciencias. Creemos que no ha calado aún suficientemente entre los historiadores el mensaje de la implicación que la aventura intelectual de las comunidades científicas tiene para la comprensión por partc de profesores y alumnos de los procesos de enseñanza y aprendizaje.

Muchas personas quedaron sorprendidas de la magnitud del congreso y de la alta cualificación de la investigación sobre historia de las ciencias y las técnicas que se realiza en España. ¿Es una moda pasajera? Parece ser que no. Desde hace más de quince años la Sociedad Española de Historia de las Ciencias trabaja en silencio agrupando a varios cientos de investigadores. Resalta, por otro lado, la escasa atención que las universidades suelen dedicar a estos temas: muy pocas licenciaturas, en nuestro país, dedican una parte de sus programas a los aspectos históricos y epistemológicos de las disciplinas. Debido a ello, muchos de los licenciados universitarios carecen de una formación globalizada sobre los contenidos, objetivos y metodologías de la carrera cursada. En todo caso, se relega al tercer ciclo (doctorado) la impartición de estas materias que deberían constituir el armazón troncal de cada titulación.

Dejando a un lado la discusión de esta situación de la historia de las ciencias en las universidades y que dejamos para otro momento, debe destacarse aquí una de las conclusiones más novedosas del congreso. En la asamblea de clausura, este XIX Congreso InternacionaI de Historia de la Ciencia ha pedido que la Ciencia, como institución mundial que pretende construir imágenes cada vez más ajustadas y racionales de nuestro mundo a través del método científico, sea declarada patrimonio de la humanidad.

Ya Roger Bacon hace varios siglos era consciente de que el objetivo de la ciencia es la «mejora de la condición humana». Hoy, más que nunca, los científicos y los historiadores de las ciencias, temen por el uso que los poderes políticos y económicos pueden hacer de sus descubrimientos. La historia muestra que la aventura de la ciencia no es un proceso neutro de búsqueda desinteresada de la verdad de la naturaleza; recientes acontecimientos muestran que las implicaciones ideologicas, cconómicas y políticas del avance científico son cada día mayores.

No sabemos si los organismos internacionales accptarán para su estudio esta propuesta del XIX Congreso Internacional de Historia de la Ciencia. Pero sí está clara la conciencia que tos asistentes teníamos del lugar del conocimiento científico en nuestra sociedad.

Leandro Sequeiros ICE de la Universidad de Zaragoza 